\title{
ONE-TO-ONE MAPPINGS
}

\author{
DIX H. PETTEY
}

\begin{abstract}
In an earlier paper, the author showed that $E^{2}$ can never be the image, under a nontopological 1-1 mapping, of a connected, locally connected, locally compact topological space. In this paper, we show that several other spaces, including $S^{2}$ and $I^{2}$ share this property with $E^{2}$.
\end{abstract}

By a mapping we will mean a continuous function. A topological space $Y$ will be said to have Property $\mathrm{H}$ if for each connected, locally connected, locally compact topological space $X$ and each 1-1 mapping $f$ of $X$ onto $Y$, $f$ is a homeomorphism.

In [1] it was shown that the Euclidean plane has Property $H$. In the present paper, we show that several other spaces, including the 2-sphere and the 2-cell, also have this property.

We will let $E^{2}, S^{2}$, and $I^{2}$ denote, respectively, the plane, the unit 2sphere, and the unit 2-cell. For an arbitrary 2-cell $K$, we will let Int $K$ and $\mathrm{Bd} K$ denote, respectively, the interior and boundary of $K$, where $K$ is regarded as a 2-manifold with boundary.

By a generalized continuum, we will mean a connected, locally compact metric space. (It follows from [3, Corollary, p. 111] that such a space is always separable.)

THEOREM 1. Let $Y$ be a locally connected, locally compact metric space having the following property: for each simple closed curve $J$ in $Y$, there is a 2-cell $K$ in $Y$ such that $J=\mathrm{Bd} K$ and such that $\mathrm{Int} K$ is an open set in $Y$. Then $Y$ has Property $\mathrm{H}$.

Proof. Assume that $Y$ does not have Property $H$. Then there is a connected, locally connected, locally compact topological space $X$ and a nontopological 1-1 mapping $f$ of $X$ onto $Y$.

By [2, Theorem 1, p. 1321], $X$ is metrizable and may therefore be regarded as a generalized continuum. It follows then from $[4,5.2$, p. 38] that $X$ is arcwise connected. Also, since $X$ is connected and $f$ is continuous,

Received by the editors July 16,1970 .

AMS 1970 subject classifications. Primary 54C05, 54C10; Secondary 57A05.

Key words and phrases. One-to-one mapping, 2-sphere, 2-cell. 
$Y$ is connected and is, therefore, a locally connected generalized continuum. Hence, by [5, Theorem 7, p. 1430] there is a topological ray $\alpha$ in $X$ such that $f(\alpha)$ is a simple closed curve in $Y$.

By our hypothesis, then, there is a 2-cell $K$ in $Y$ such that $f(\alpha)=\mathrm{Bd} K$ and such that Int $K$ is an open set in $Y$. We shall show that $f^{-1}(K)$ is connected, locally connected, and locally compact.

Let $O=X-f^{-1}(K)$ and let $U=f^{-1}($ Int $K)$. Then $O$ and $U$ are disjoint open sets in $X$ and $f^{-1}(K)=\alpha \cup U$. To show that $f^{-1}(K)$ is connected, we observe that $X-\alpha=O \cup U$. Since each of $X$ and $\alpha$ is connected, we have $\alpha \cup U\left(=f^{-1}(K)\right)$ connected. A similar argument shows that $f^{-1}(K)$ is locally connected. For if $x \in f^{-1}(K)$ and $V$ is an open set in $X$ with $x \in V$ then there is a connected open set $W$ in $X$ such that $x \in W \subset V$ and such that $W \cap \alpha$ is connected. Since $W \cap O$ and $W \cap U$ are disjoint open sets and $W-\alpha=(W \cap O) \cup(W \cap U)$, we have $(W \cap \alpha) \cup(W \cap U)$ connected; i.e., $W \cap f^{-1}(K)$ is a connected set. The local compactness of $f^{-1}(K)$ is an immediate consequence of the local compactness of $X$ and the fact that $f^{-1}(K)$ is a closed subset of $X$. Thus, $f^{-1}(K)$ is connected, locally connected, and locally compact.

Now, letting $[0,1)$ denote the real-line interval $\{t \mid 0 \leqq t<1\}$, define $X^{\prime}$ and $Y^{\prime}$ to be subspaces of $X \times[0,1)$ and $Y \times[0,1)$, respectively, as follows:

and

$$
X^{\prime}=\left(f^{-1}(K) \times\{0\}\right) \cup(\alpha \times[0,1))
$$

$$
Y^{\prime}=(K \times\{0\}) \cup(B d K \times[0,1)) .
$$

Then $X^{\prime}$ is connected, locally connected, and locally compact; and $Y^{\prime}$ is homeomorphic to $E^{2}$. Define a function $g$ from $X^{\prime}$ onto $Y^{\prime}$ by letting $g(x, t) \doteq(f(x), t)$ for each $(x, t) \in X^{\prime}$. Since $f$ is 1-1 and continuous, $g$ is $1-1$ and continuous. Therefore, it follows from [1, Theorem 4.4, p. 308] that $g$ is a homeomorphism. But this implies that $f \mid \alpha$ is a homeomorphism of $\alpha$ onto $\mathrm{Bd} K$. Since $\alpha$ is a topological ray and $\mathrm{Bd} K$ is a simple closed curve we have a contradiction and the proof is complete.

Each of the following corollaries is an immediate consequence of Theorem 1.

Corollary 1.1. $S^{2}$ has Property H.

CoRollary 1.2. $I^{2}$ has Property $\mathrm{H}$.

COROLlary 1.3. If $Y$ is a simply connected, locally connected, locally compact subspace of $E^{2}$, then $Y$ has Property $\mathrm{H}$.

It follows from Theorem 1 that if a topological space $Y$ is the union of two 2-spheres which have exactly one point in common, then $Y$ has Property H. Our next theorem gives us a more general result of this nature. 
THEOREM 2. Suppose that a topological space $Y$ is the union of two closed subspaces $Y_{1}$ and $Y_{2}$ such that (1) $Y_{1}$ and $Y_{2}$ have exactly one point in common, and (2) each of $Y_{1}$ and $Y_{2}$ has Property $H$. Then $Y$ has Property $\mathrm{H}$.

Proof. Suppose that $X$ is a connected, locally connected, locally compact topological space and that $f$ is a 1-1 mapping of $X$ onto $Y$. Let $q$ denote the intersection of $Y_{1}$ and $Y_{2}$ and let $X_{1}, X_{2}$, and $p$ denote, respectively, $f^{-1}\left(Y_{1}\right), f^{-1}\left(Y_{2}\right)$, and $f^{-1}(q)$. Then $Y_{1}-q$ and $Y_{2}-q$ are disjoint open sets in $Y$. Consequently, $X_{1}-p$ and $X_{2}-p$ are disjoint open sets in $X$. Since, $X$ is connected, this implies that each of the sets $X_{1}$ and $X_{2}$ is connected. A similar argument shows that for each connected open neighborhood $N$ of $p$ in $X$, each of the sets $N \cap X_{1}$ and $N \cap X_{2}$ is connected. Thus, each of $X_{1}$ and $X_{2}$ is locally connected at $p$ and, therefore, locally connected. Since each of the sets $Y_{1}$ and $Y_{2}$ is closed in $Y$, each of $X_{1}$ and $X_{2}$ is a closed subset of the locally compact space $X$ and is therefore locally compact. Because each of $Y_{1}$ and $Y_{2}$ has Property $\mathrm{H}$, it now follows that $f \mid X_{1}$ is a homeomorphism of $X_{1}$ onto $Y_{1}$ and $f \mid X_{2}$ is a homeomorphism of $X_{2}$ onto $Y_{2}$. Consequently, $f$ is a homeomorphism of $X$ onto $Y$.

\section{REFERENCES}

1. Dix H. Pettey, Mappings onto the plane, Trans. Amer. Math. Soc. 157 (1971), 297-309.

2. V. V. Proizvolov, One-to-one mappings onto metric spaces, Dokl. Akad. Nauk SSSR 158 (1964), 788-789 = Soviet Math. Dokl. 5 (1964), 1321-1322. MR 30 \#5276.

3. W. Sierpiński, Sur les espaces métriques localement séparables, Fund. Math. 21 (1933), 107-113.

4. G. T. Whyburn, Analytic topology, 2nd ed., Amer. Math. Soc. Colloq. Publ., vol. 28, Amer. Math. Soc., Providence. R.I., 1963. MR 32 \#425.

5. - On compactness of mappings, Proc. Nat. Acad. Sci. U.S.A. 52 (1964), 1426-1431. MR 31 \#722.

Department of Mathematics, University of Missouri, Columbia, Missouri 65201 